\title{
Accurate random-phase approximation calculation of low-lying states on a three-dimensional Cartesian mesh
}

\author{
H. Imagawa and Y. Hashimoto \\ Institute of Physics, University of Tsukuba, Ibaraki 305-8571, Japan
}

(Received 7 October 2002; published 14 March 2003)

\begin{abstract}
We present a simple and efficient method for calculating excitation energies and transition probabilities of low-lying states described by Hartree-Fock (HF) plus random-phase approximation (RPA) with Skyrme force. The method employs conjugate gradient method to solve the RPA equations in the mixed representation of coordinate and occupied orbitals, which was proposed recently. To obtain accurate results with coarse mesh (1 $\mathrm{fm}$ ) calculation, we find a useful prescription. Performing self-consistently three-dimensional Cartesian mesh calculation with Lagrange mesh method in solving HF and RPA equations, we take an average of quantities calculated with even and odd mesh points in one direction. As a demonstration of our method, we show the numerical results of energies for spurious mode of translation of ${ }^{16} \mathrm{O}$ and the excitation energies and reduced transition probabilities for first $3^{-}$state of ${ }^{16} \mathrm{O}$ and ${ }^{208} \mathrm{~Pb}$.
\end{abstract}

DOI: 10.1103/PhysRevC.67.037302

PACS number(s): 21.60.Jz

The Skyrme Hartree-Fock (SHF) method [1] has been developed with the aim of describing nuclear ground states for the last several decades. The SHF method combined with the BCS method is known to well reproduce binding energies, charge radii, and deformations throughout the Periodic Table $[2,3]$. As for the excited states, random-phase approximation (RPA) with Skyrme interaction has been used in the description of giant resonance [4] and low-lying states [5-7].

In calculating the SHF, Bonche et al. [8] showed that the three-dimensional (3D) Cartesian mesh calculation is suitable to describe nuclear deformations. Baye and Heenen [9] proposed Lagrange mesh method which provides an explanation for the unexpected accuracy of Hartree-Fock calculations performed on the 3D Cartesian mesh.

Recently, Muta et al. [10] reported a computational method for solving the RPA equations in the coordinate representation on 3D Cartesian mesh. They succeeded in calculating a few low-lying states of deformed nuclei with a simplified local effective interaction. In their method, the RPA equations are given in a mixed representation of coordinate, instead of unoccupied orbitals, and occupied orbitals. The method has the following two merits. One is that 3D Cartesian mesh calculation can be performed self-consistently in solving both HF and RPA equations. The other is that the unoccupied states are not necessary in the procedure, i.e., no truncation of the unoccupied orbitals is required. However, their method has two difficulties. The first point is that fine mesh size is required in their calculation in order to separate physical modes from spurious zero modes. The second point is that imaginary eigenvalues of the RPA equations cannot be treated with their method.

In this paper, we improve their formulation to be capable of treating imaginary eigenvalues of the RPA equations. Making the best use of the improved formulation, we propose a simple method for estimating values close to converged ones in a coarse mesh calculation. We apply the method to the calculation of total energies, spurious states of ${ }^{16} \mathrm{O}$ and the first $3^{-}$state of ${ }^{16} \mathrm{O}$ and ${ }^{208} \mathrm{~Pb}$, and compare our numerical results with the ones of Ref. [6]. Though our method can be applied to deformed nuclei, we restrict our- selves to the spherical nuclei for the purpose of comparison with other methods.

Here we briefly explain the formulation of Ref. [10]. The RPA equations in a mixed representation of coordinate and occupied orbitals, which were first derived by Lemmer and Vénéroni [11], are

$$
\begin{gathered}
\sum_{j x^{\prime}} A_{i j}\left(x, x^{\prime}\right) X_{j}^{\nu}\left(x^{\prime}\right)+B_{i j}\left(x, x^{\prime}\right) Y_{j}^{\nu}\left(x^{\prime}\right)=\hbar \omega_{\nu} X_{i}^{\nu}(x), \\
\sum_{j x^{\prime}} B_{i j}^{*}\left(x, x^{\prime}\right) X_{j}^{\nu}\left(x^{\prime}\right)+A_{i j}^{*}\left(x, x^{\prime}\right) Y_{j}^{\nu}\left(x^{\prime}\right)=-\hbar \omega_{\nu} Y_{i}^{\nu}(x),
\end{gathered}
$$

where $\hbar \omega_{\nu}$ denotes the eigenvalue for the eigenmode $\nu$, the subscripts $i$ and $j$ represent occupied states, and $x$ stands for a set of space coordinate $\boldsymbol{r}$, spin $\sigma$, and isospin $\tau$. We use the notation $\Sigma_{x} \equiv \Sigma_{\sigma \tau} \int d \boldsymbol{r}$. We rewrite Eq. (1) as

$$
\left(\begin{array}{cc}
A & B \\
B^{*} & A^{*}
\end{array}\right)\left(\begin{array}{l}
X \\
Y
\end{array}\right)_{\nu}=\hbar \omega_{\nu}\left(\begin{array}{cc}
1 & 0 \\
0 & -1
\end{array}\right)\left(\begin{array}{l}
X \\
Y
\end{array}\right)_{\nu} .
$$

As pointed out in Ref. [10], Eq. (2) can be solved by means of the conjugate gradient method (CGM) [12], which is an efficient iterative method for calculating a few real eigenvalues and eigenvectors for a generalized eigenvalue equation. The solution with the lowest eigenvalue of Eq. (2) usually corresponds to one of the spurious modes. Making use of the Schmidt orthogonalization method, we can obtain excited states in order of increasing eigenvalue.

There is a possibility that the above formulation cannot be implemented when spatial symmetries or constraints are imposed. For example, if constraints for center of mass and principal axes are imposed in the HF calculation, eigenvalues corresponding to zero modes of translation or rotation may be imaginary in RPA calculation. Then CGM cannot be used for RPA calculation, because one of the conditions for the application of the CGM that all of the eigenvalues of generalized eigenvalue equation are real is not satisfied in the case. 


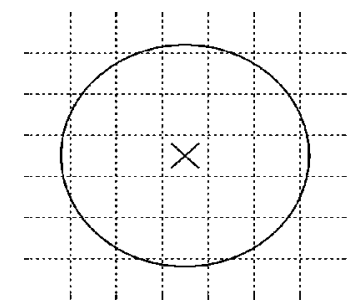

(a) even

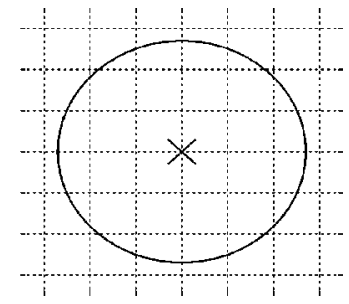

(b) odd
FIG. 1. Schematic illustration of two ways of setting the mesh on space. The intersection points of broken lines represent mesh points. Each circle represents a nucleus, whose center of mass is marked by a cross $(X)$. The center of mass of a nucleus is located at the middle point of the mesh points in (a) and coincides with a mesh point in (b).

In order to impose spatial symmetries in the calculation, which will be necessary in our method to obtain accurate solution with a coarse mesh calculation, we improve the formulation so that we can treat the imaginary eigenvalue of the RPA equations. We introduce quantities $\phi_{i}^{( \pm) \nu}(x)=\left[X_{i}^{\nu}(x)\right.$ $\left.\pm Y_{i}^{\nu *}(x)\right] / \sqrt{2}$, and we express the RPA equations in this representation as

$$
\left(\begin{array}{cc}
A & \pm B \\
\pm B^{*} & A^{*}
\end{array}\right)\left(\begin{array}{c}
\phi^{( \pm)} \\
\phi^{( \pm) *}
\end{array}\right)_{\nu}=\hbar \omega_{\nu}\left(\begin{array}{c}
\phi^{(\mp)} \\
\phi^{(\mp) *}
\end{array}\right)_{\nu}
$$

These equations correspond to the RPA equations in $P-Q$ representation [13]. We then write equations for $\phi_{i}^{(-) \nu}(x)$ only,

$$
\begin{gathered}
\left(\begin{array}{cc}
A & -B \\
-B^{*} & A^{*}
\end{array}\right)\left(\begin{array}{cc}
A & B \\
B^{*} & A^{*}
\end{array}\right)\left(\begin{array}{cc}
A & -B \\
-B^{*} & A^{*}
\end{array}\right)\left(\begin{array}{c}
\phi^{(-)} \\
\phi^{(-)} *
\end{array}\right)_{\nu} \\
=\left(\hbar \omega_{\nu}\right)^{2}\left(\begin{array}{cc}
A & -B \\
-B^{*} & A^{*}
\end{array}\right)\left(\begin{array}{c}
\phi^{(-)} \\
\phi^{(-)} *
\end{array}\right)_{\nu}
\end{gathered}
$$

Appling the CGM for Eq. (4), we can obtain solution corresponding to negative eigenvalue $\left(\hbar \omega_{\nu}\right)^{2}$ of Eq. (4), which means pure imaginary energy $\hbar \omega_{\nu}$.

We next explain how we can obtain accurate excitation energies with a rather coarse mesh calculation. We perform the RPA calculations on two types of 3D Cartesian meshes (see Fig. 1) with even and odd mesh points in one-direction, and we take an average of the two numerical results such as excitation energies and transition probabilities. We use the Lagrange mesh method [9] in order to approximate firstorder and second-order differential operators. As is shown, in the practical examples below, this procedure provides us with accurate results even when we employ rather coarse mesh size.

In both meshes of Figs. 1(a) and 1(b), we employ points inside a cube. The center of mass of the nucleus is constrained to locate at the center of the cube. Hereafter, we call the mesh setting in Fig. 1(a) the even mesh points case, and in Fig. 1(b) the odd mesh points case. To diminish numerical efforts, we impose symmetries with respect to reflection in $x y, y z$, and $z x$ planes (the point group $D_{2 h}$ ) on density in

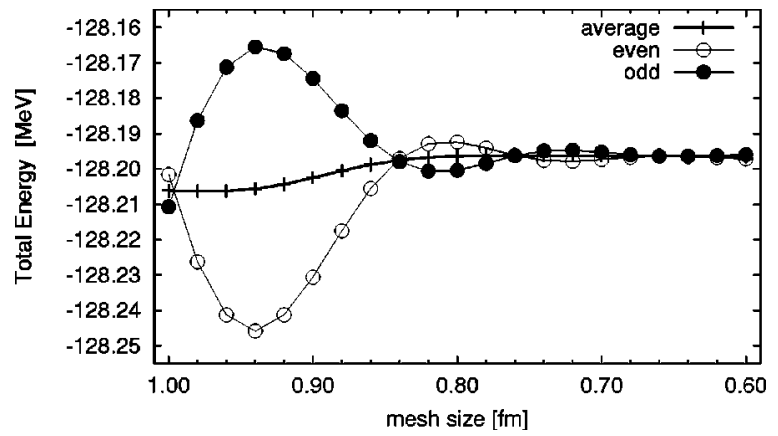

FIG. 2. Mesh size dependence of HF energies of ${ }^{16} \mathrm{O}$. Open (filled) circles denote numerical results of the even (odd) mesh points case where $30^{3}\left(31^{3}\right)$ mesh points are used. Plus signs $(+)$ are averages of the even and the odd mesh points cases at each mesh size.

the HF calculation [8], which allows triaxial deformation. The same symmetris are imposed on transition densities in RPA calculation [14]. In other words, the transition densities are even or odd with respect to reflection of $x y, y z$, and $z x$ plane. The solutions of the RPA equations are then classified into eight groups.

For the effective interaction, we use SIII force [15]. The original three-body term is changed into a density dependent two body term [16]. In the Hamiltonian density, we include both time-even and time-odd components [17].

We apply our method to two spherical nuclei, ${ }^{16} \mathrm{O}$ and ${ }^{208} \mathrm{~Pb}$, as illustrative examples. In HF and RPA calculations for ${ }^{16} \mathrm{O}$, we fix the number of the mesh points to $30^{3}$ (even mesh points case) and $31^{3}$ (odd mesh points case). The calculations are carried out with the mesh size being changed from $1.0 \mathrm{fm}$ to $0.6 \mathrm{fm}$ by step-size $0.02 \mathrm{fm}$.

In Fig. 2, we show the HF energies of ${ }^{16} \mathrm{O}$. As is seen from the figure, the total energy is rather different between the results in the even and the odd mesh points cases. As the mesh size goes smaller, HF energies of both the even and the odd mesh points cases converge to a value $-128.20 \mathrm{MeV}$. If we take average of the even and the odd mesh points cases, the converged value can be obtained for all the mesh size ranging from 1 to $0.6 \mathrm{fm}$. The fluctuation of the average is very small, within $10 \mathrm{keV}$.

In Fig. 3, we show the squared energies $\left(\hbar \omega_{0}\right)^{2}$ of the spurious modes of translation for ${ }^{16} \mathrm{O}$. In the figure, we can

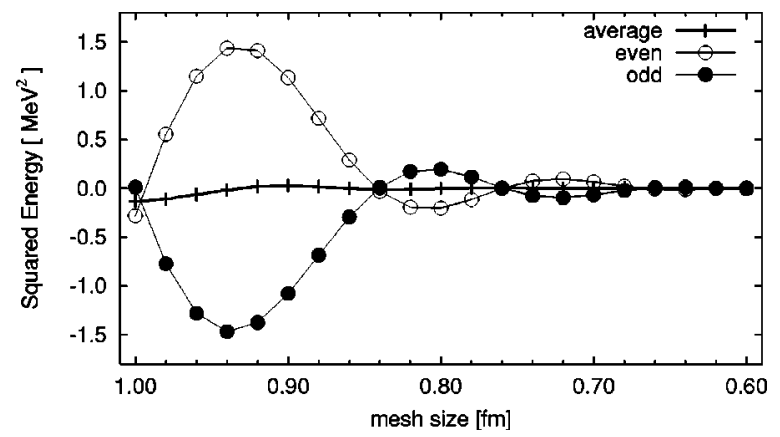

FIG. 3. Mesh size dependence of calculated squared energies for spurious modes of ${ }^{16} \mathrm{O}$. Notations are the same as in Fig. 2. 


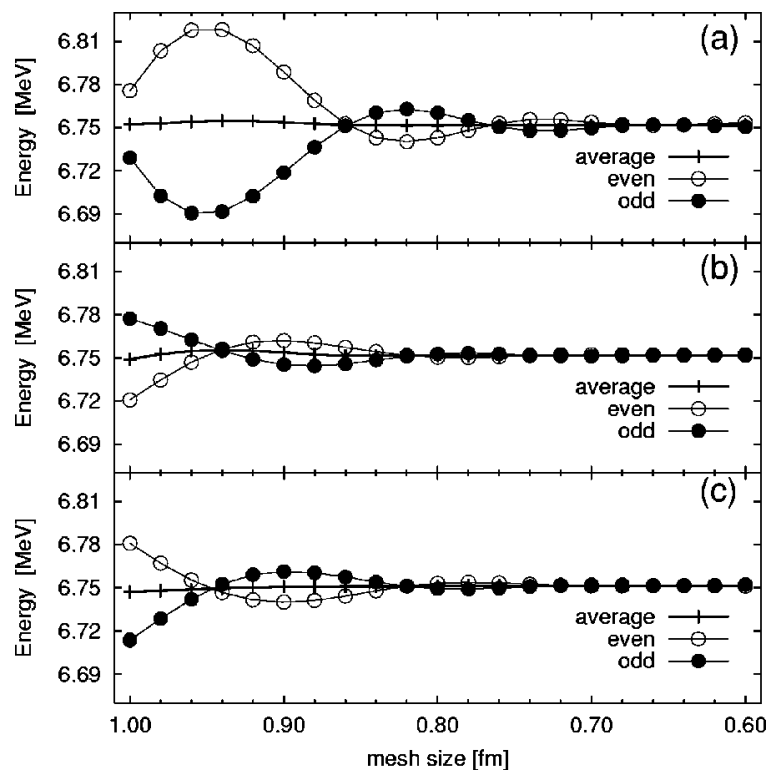

FIG. 4. Mesh size dependence of excitation energies for the first $3^{-}$state of ${ }^{16} \mathrm{O}$. Notations are the same as in Fig. 2. Energy levels for $3^{-}$state with sevenfold degeneracy split into three groups (a), (b), and (c) because of symmetry of 3D Cartesian mesh. Each of (a) and (b) has threefold degeneracy and (c) has one component.

again see the strong mesh size dependence of the squared energies in accordance with that in Fig. 2: There is a large difference between the results in the even and the odd mesh points cases. As the mesh size goes smaller, the squared energies of both the even and the odd mesh points cases converge to zero. The average of the even and the odd mesh points cases is close to the converged value for all over the displayed mesh size. The fluctuation of the average is within $0.2 \mathrm{MeV}^{2}$, even with a mesh size of $1 \mathrm{fm}$.

In Figs. 4 and 5, we show the excitation energies and the reduced electric-octupole transition probabilities for first $3^{-}$ state of ${ }^{16} \mathrm{O}$. Because of the symmetries with 3D Cartesian mesh, seven degenerate eigenvalues of $3_{1}^{-}$state split into the following three groups. In the first group (a), there are three components, where each transition probability is proportional to $r^{3} Y_{30} \delta \rho \sim z\left(5 z^{2}-3 r^{2}\right) \delta \rho$, and its permutations of $x, y$ and $z$, i.e., $x\left(5 x^{2}-3 r^{2}\right) \delta \rho$ and $y\left(5 y^{2}-3 r^{2}\right) \delta \rho$. In the second group (b) there are three components, where each transition probability is proportional to $r^{3}\left(Y_{32}+Y_{3-2}\right) \delta \rho$ $\sim z\left(y^{2}-x^{2}\right) \delta \rho$ and its permutations of $x, y$, and $z$. In the third group (c) there is one component, where transition probability is proportional to $r^{3}\left(Y_{32}-Y_{3-2}\right) \delta \rho \sim x y z \delta \rho$. In Fig. 4 (Fig. 5), as the mesh size goes smaller, excitation energies (reduced transition probabilities) of the even and the odd mesh points cases converge to a value $6.75 \mathrm{MeV}(6.93$ Weisskopf unit). In all of the cases of (a), (b), and (c), the averages of the results in the even and the odd mesh points cases are close to the converged value for all mesh size in the figures. The fluctuation of the averages is within $10 \mathrm{keV}$ (0.04 Weisskopf unit). In Blaizot and Gogny's calculation [6], where the RPA equations in particle-hole representation were diagonalized in an spherical harmonic oscillator basis,

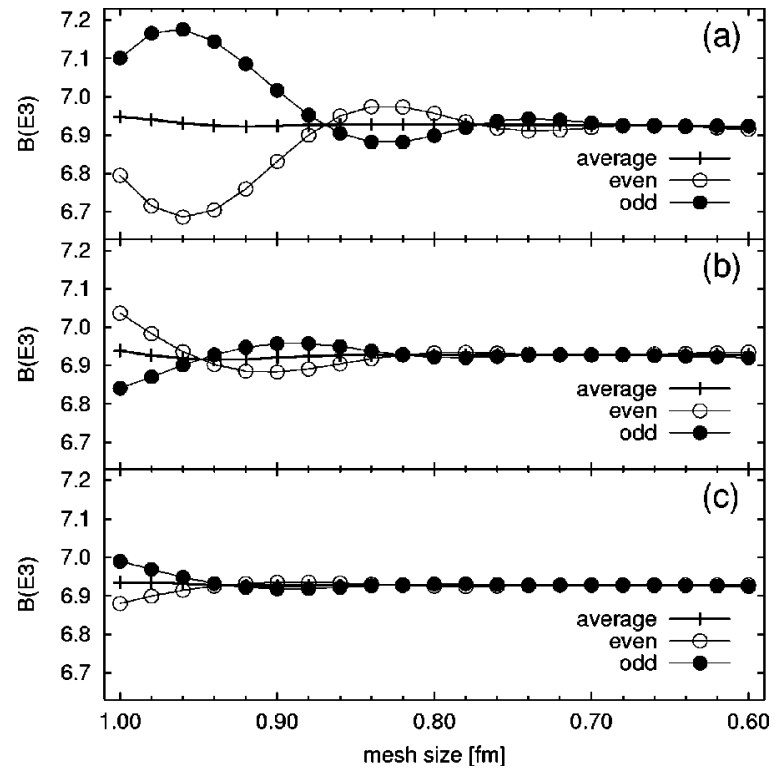

FIG. 5. Mesh size dependence of reduced electric-octupole transition probabilities $B\left(E 3 ; 3_{1}^{-} \rightarrow 0_{1}^{+}\right)$of ${ }^{16} \mathrm{O}$ in Weisskopf unit. The notations are the same as in Fig. 4.

the excitation energy is $6.77 \mathrm{MeV}$ and the reduced transition probability is 6.23 Weisskopf unit. The excitation energy of their calculation coincides accurately with ours, but the reduced transition probability of their calculation is rather smaller than our result. Blaizot and Gogny pointed out that it is necessary to use a large particle-hole configuration space in order to get reliable values of the transition probabilities for their calculation. Although we do not understand clearly the origin of the difference, one of the possible origin is the fact that our calculation does not impose any truncation on the particle orbitals, whereas Blaizot and Gogny did.

In order to illustrate the feasibility of our method in the heavier nuclei, we show the numerical results of excitation energies and the reduced electric-octupole transition probabilities for the first $3^{-}$state of ${ }^{208} \mathrm{~Pb}$ in Table I. The calculations are achieved with four different settings of mesh size and box size, i.e., (i) large mesh size and small box size, (ii) small mesh size and small box size, (iii) large mesh size and large box size, and (iv) small mesh size and large box size. The converged value of excitation energy (reduced transition probability) obtained from the calculation is $2.245 \mathrm{MeV}$ (44.1 Weisskopf unit). The computational size is the smallest for the calculation (i). Even in this calculation, the averages for the excitation energies and reduced transition probabilities of the even and the odd mesh points cases are about 2.2 $\mathrm{MeV}$ and about 44 Weisskopf unit, respectively. Thus, we conclude that our simple method of averaging gives us the converged excitation energy and transition strength even with the coarse mesh size of $1 \mathrm{fm}$ and small box size of $(20 \mathrm{fm})^{3}$ is used. In Blaizot and Gogny's calculation [6], the excitation energy is $2.82 \mathrm{MeV}$ and the reduced transition probability is 33 Weisskopf unit. The excitation energy of their calculation is somewhat larger than that of our calculation and the reduced transition probability of their calcula- 
TABLE I. Excitation energy and reduced transition probability of first $3^{-}$state of ${ }^{208} \mathrm{~Pb} . \Delta h$ is mesh size. $N$ is the number of mesh points of the edge of the cubic box. $V$ is volume of cubic box. "av." in the column of $N$ represents average value of upper two lines of "even" and "odd" cases. (a), (b), and (c) are the same as in Figs. 4 and 5.

\begin{tabular}{|c|c|c|c|c|c|c|c|c|c|}
\hline & \multirow{2}{*}{$\begin{array}{c}\Delta h \\
(\mathrm{fm})\end{array}$} & \multirow[t]{2}{*}{$N$} & \multirow{2}{*}{$\begin{array}{c}V \\
\left(\mathrm{fm}^{3}\right)\end{array}$} & \multicolumn{3}{|c|}{ Energy $(\mathrm{MeV})$} & \multicolumn{3}{|c|}{$B(E 3)$ (W.u.) } \\
\hline & & & & (a) & (b) & (c) & (a) & (b) & (c) \\
\hline & & 19 & $19.00^{3}$ & 2.140 & 2.224 & 2.205 & 45.4 & 44.4 & 44.5 \\
\hline \multirow[t]{3}{*}{ (i) } & 1.00 & 20 & $20.00^{3}$ & 2.302 & 2.246 & 2.263 & 41.6 & 44.2 & 43.9 \\
\hline & & av. & & 2.221 & 2.235 & 2.234 & 43.5 & 44.3 & 44.2 \\
\hline & & 24 & $19.20^{3}$ & 2.263 & 2.253 & 2.264 & 43.7 & 43.8 & 43.8 \\
\hline \multirow[t]{3}{*}{ (ii) } & 0.80 & 25 & $20.00^{3}$ & 2.224 & 2.233 & 2.226 & 44.0 & 44.4 & 44.4 \\
\hline & & av. & & 2.243 & 2.243 & 2.245 & 43.8 & 44.1 & 44.1 \\
\hline & & 29 & $29.00^{3}$ & 2.191 & 2.246 & 2.240 & 45.8 & 44.0 & 44.1 \\
\hline \multirow[t]{3}{*}{ (iii) } & 1.00 & 30 & $30.00^{3}$ & 2.290 & 2.238 & 2.250 & 42.0 & 44.4 & 44.2 \\
\hline & & av. & & 2.240 & 2.242 & 2.245 & 43.9 & 44.2 & 44.1 \\
\hline & & 39 & $29.25^{3}$ & 2.246 & 2.245 & 2.245 & 44.1 & 44.1 & 44.1 \\
\hline \multirow[t]{2}{*}{ (iv) } & 0.75 & 40 & $30.00^{3}$ & 2.245 & 2.246 & 2.245 & 44.2 & 44.1 & 44.1 \\
\hline & & av. & & 2.245 & 2.245 & 2.245 & 44.1 & 44.1 & 44.1 \\
\hline
\end{tabular}

tion is considerably smaller than that of our calculation. We do not understand the origin of this discrepancy. Since we noticed a sizable difference in the transition strength even for light nucleus ${ }^{16} \mathrm{O}$, one may expect a difficulty to obtain converged results in the calculation of particle-hole basis. We would like to point out that our results presented here is close to converged values.

In summary, we have shown a simple and efficient method for accurately calculating low-lying states properties of nuclei in the framework of HF and RPA with Skyrme force. There are two essential points in our method: The first is that, in solving the HF and RPA equations we have selfconsistently performed the 3D Cartesian mesh calculation with Lagrange mesh method. We have checked that our method is less successful if a finite difference method (ninepoint formula) is used instead of the Lagrange mesh method. The second is that we have taken the average of the quantities calculated with the even and the odd mesh points in one direction. Then even with the mesh size of $1 \mathrm{fm}$, which is often employed in the ground state calculation, we can obtain accurate results of excitation energies and reduced transition probabilities. We have demonstrated the feasibility of the method in the calculations of HF energies, spurious modes, the excitation energies, and reduced transition probabilities for first $3^{-}$state of ${ }^{16} \mathrm{O}$ and ${ }^{208} \mathrm{~Pb}$ with Skyrme SIII force. Although they are spherical nuclei, our method is readily applicable to deformed nuclei. The systematic analyses of the other kinds of low-lying excited states in the spherical as well as deformed nuclei will be given in succeeding papers.

The authors are grateful to Professor Yabana for continuous encouragement and stimulating discussions. They would like to thank Professor Matsuyanagi and Professor Sakata for encouragement and fruitful suggestions. They also thank Professor Tajima, Dr. Mizutori, Dr. Nakatsukasa, and Dr. Hagino for valuable discussions and comments. The computational calculations were performed by YITP, Kyoto University.
[1] D. Vautherin and D. M. Brink, Phys. Rev. C 5, 626 (1972).

[2] N. Tajima, S. Takahara, and N. Onishi, Nucl. Phys. A603, 23 (1996).

[3] S. Goriely, F. Tondeur, and J. Pearson, At. Data Nucl. Data Tables 77, 311 (2001)

[4] Nguyen Van Giai and N. Sagawa, Nucl. Phys. A371, 1 (1981).

[5] S. Krewald et al., Nucl. Phys. A281, 166 (1977).

[6] J. P. Blaizot and D. Gogny, Nucl. Phys. A284, 429 (1977).

[7] M. Waroquier, G. Wenes, and K. Heyde, Nucl. Phys. A404, 298 (1983).

[8] P. Bonche et al., Nucl. Phys. A443, 39 (1985).
[9] D. Baye and P. H. Heenen, J. Phys. A 19, 2041 (1986).

[10] A. Muta et al., Prog. Theor. Phys. 108, 1065 (2002).

[11] R. H. Lemmer and M. Veneroni, Phys. Rev. 170, 883 (1968).

[12] W. W. Bradbury and R. Fletcher, Numer. Math. 9, 259 (1966).

[13] D. J. Row, Nuclear Collective Motion (Methuen, London, 1970).

[14] H. Imagawa and Y. Hashimoto (in preparation).

[15] M. Beiner et al., Nucl. Phys. A238, 29 (1975).

[16] B. D. Chang, Phys. Lett. 56B, 205 (1975).

[17] Y. M. Engel et al., Nucl. Phys. A249, 215 (1975). 